\title{
The Feasibility of an Ex-vivo Sentinel Lymph Mapping Using Preoperative Radioisotope Injection in Cases of Extraperitoneal Rectal Cancer
}

\author{
Jun Seok Park, Gyu-Seog Choi, Hye Jin Kim, Soo Yeon Park, Yun Jung Park, Sang-Woo Lee', Ziguang Xu², \\ Han Ik Bae ${ }^{2}$ \\ Departments of Surgery, ${ }^{1}$ Nuclear Medicine, and ${ }^{2}$ Pathology, Kyungpook National University Hospital, Kyungpook National University School \\ of Medicine, Daegu, Korea
}

Purpose: The purpose of this research was to evaluate the feasibility of sentinel lymph node (SLN) mapping involving transanal injection with an ex-vivo mapping in patients with rectal cancer.

Methods: Between April 2007 and December 2009, 20 consecutive patients with T1-3, N0-1 clinical stage rectal cancer preoperatively underwent a SLN procedure using submucosal ${ }^{99 \mathrm{~m}} \mathrm{Tc}$-phytate injection. All the patients underwent a total mesorectal excision. After the standard surgical resection, all specimens were identified on lymphoscintigraphy, and bench work was done to pick up the sentinel node basin. All the lymph nodes (non-SLNs and SLNs) were examined using conventional hematoxylin and eosin staining and immunohistochemistry with anti-cytokeratin antibodies.

Results: SLNs were identified from 19 of 20 patients with rectal cancer. The total number of sentinel nodes retrieved from the surgical specimens was 29, and the mean number per patient was 1.6 (range, 0 to 4 ). In three patients, the SLN was the only positive lymph node. There was one false-negative case with a sensitivity of $88.8 \%$ and two upstaged cases (20.0\%). The SLN samples from rectal cancer are mainly localized in the pararectal region, but aberrant nodes receive direct drainage from the rectal cancer. On planar lymphoscintigraphy, $15.7 \%$ of all patients had aberrant lymphatic drainage to the sigmoid mesenteric or sigmoid lymph node station.

Conclusion: In conclusion, the intraoperative transanal injection for ex-vivo SLN navigation is a safe, feasible surgical modality in patients with rectal cancer. Large studies are warranted to determine the clinical significance of the SLN concept and micrometastasis in rectal cancer.

Keywords: Rectal neoplasms; Transanal injection; Sentinel lymph node; Lymphoscintigraphy

\section{INTRODUCTION}

Lymph node involvement is one of the most important prognostic factors in the evaluation of colorectal cancer. Randomized trials have demonstrated a $40 \%$ reduction in recurrence and a $33 \%$

Received: February 24, 2011 Accepted: April 13, 2011

Correspondence to: Gyu-Seog Choi, M.D.

Department of Surgery, Kyungpook National University School of Medicine, 50 Samdeok-dong 2-ga, Jung-gu, Daegu 700-721, Korea

Tel: +82-53-420-5619, Fax: +82-53-421-0510

E-mail: kyuschoi@mail.knu.ac.kr

(c) 2011 The Korean Society of Coloproctology

This is an open-access article distributed under the terms of the Creative Commons Attribution NonCommercial License (http://creativecommons.org/licenses/by-nc/3.0) which permits unrestricted non-

commercial use, distribution, and reproduction in any medium, provided the original work is properly cited. improvement in survival with the use of adjuvant chemotherapy [1]. In patients with node-negative disease, however, the benefit of adjuvant chemotherapy remains controversial although the recurrence and the mortality have been reported to reach $30 \%[2,3]$. Relapse is presumed to occur in patients who have lymph node metastasis that has not identified by conventional histopathologic examination.

The term "sentinel node" was coined in 1977 by Cabanas [4] in his study of penile carcinomas. Nowadays, sentinel lymph node (SLN) mapping is the standard nodal staging technique in patients with breast cancer. The advantage of this technique is that there is no potentially harmful auxiliary dissection. In patients with colorectal cancer, this is not an issue. Thus, the benefit of SLN mapping has to be found in fact that with this technique, potentially highrisk patients, who could develop metastases in time, can be iden- 
tified and, maybe, benefit from adjuvant therapy (upstaging to node positive level). In the majority of patients, the importance of SLN mapping will rest in this techniques ability to more accurately identify the node most likely to harbor metastasis and allow focused evaluation of that node. Focused evaluation will result in more precise prognostic information and will identify a group of patients who can likely benefit from adjuvant treatment. A number of studies have investigated the SLN mapping technique and have described successful SLN identification in colorectal cancer, with identification rates of $94-100 \%$ and upstaging ranging from 20 to $30 \%$ [5-7].

The optimal method for detecting the SLNs in patients with colorectal cancer remains controversial, and several methods, including blue dye, lymphoscintigraphy using radiocolloids, and portable gamma cameras or near-infrared fluorescence light, have been described [8]. In the majority of published studies of SLN mapping in the treatment of colorectal cancer, subserosal in-vivo injection of visible dye (i.e., methylene blue or isosulfan blue) and intraoperative identification were used. Although promising results would be expected in the colon cancer with this technique, it might be less applicable for rectal cancer than for colon cancer. Because the rectum is anatomically quite different from the colon, with a bulkier and infraperitoneal location, the conventional procedure (intraopertive, subserosal, and circumferential injection) is more difficult in the rectum. The aim of this study was to evaluate the feasibility of SLN mapping based on a combination of injection of a radioisotope prior to rectal dissection and ex-vivo SLN mapping in patients with rectal cancer and its potential application in practice.

\section{METHODS}

From May 2009 to December 2010, the prospectively collected records of 20 consecutive rectal cancer patients with T1-3, N0-1 clinical stage cancer were reviewed retrospectively. Preoperative evaluation included colonoscopy, abdominal computed tomography, magnetic resonance imaging, and blood test. Patients with rectal cancer located intraperitoneally (above peritoneal reflection), lateral node involvement confirmed by an imaging study, tumor invasion of an adjacent organ (T4), and distant metastasis and patients refusing to participate were not included in the study.

In the current study, the primary endpoints (outcome measures) are the detection rate and the sensitivity of SLN mapping in the treatment of rectal cancer, and the secondary endpoint (outcome measure) is the upstaging rate of an ex-vivo approach and assessment of the aberrant lymphatic drainage pattern. Patient recruitment and sample collection were performed in accordance with the protocol guidelines of the Institutional Review Board (IRB) of our institution, and all patients included in this study submitted a written informed consent.

The SLN procedure is illustrated in Fig. $1 .{ }^{99 \mathrm{~m}} \mathrm{Tc}$-phytate agent (0.5-1.0 mL, $1.0 \mathrm{mCi}$, non-filtered) was intraoperatively injected
Intra-operative transanal submucosal injection of ${ }^{99 m} \mathrm{Tc}$-phytate (1.0 mCi, 0.5-1.0 mL)

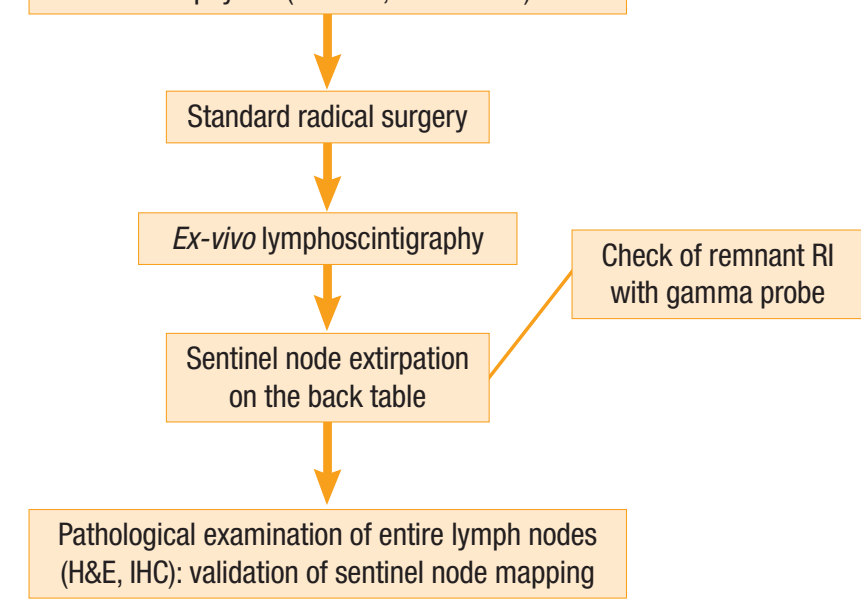

Fig. 1. Protocol of sentinel node mapping. Intraoperative ${ }^{99 \mathrm{~m}} \mathrm{Tc}-$ phytate was injected into the rectal submucosa.

RI, radioisotope; H\&E, hematoxylin and eosin staining; IHC, immunohistochemistry.

into the rectal submucosa transanally. Immediately after general anesthesia and subsequent positioning, the investigator used a Park's anal retractor and a 25-gauge spinal needle to inject a radioactive contrast medium into the areas adjacent to the tumor at two to four points, depending on the size of the tumor. After submucosal injection, all patients underwent a standard total mesorectal excision, and this included the total mesocolon of the corresponding vascular trunk. After standard surgical resection, all specimens were identified by using lymphoscintigraphy. The location and the number of sentinel nodes were recorded. Following an imaging study, bench work was done at a separate back table to pick up the sentinel basin (Fig. 2). All nodal areas were localized with a handheld gamma probe (Neo 2000; Neoprobe, Dublin, OH, USA) to confirm the radiation "hot spots," which had already been identified on lymphoscintigraphy. A lymph node was considered to be a sentinel node when it showed ex-vivo radioactive counts tenfold higher than the background radioactivity. An SLN mapping was generally performed within four hours, but no later than eight hours after the specimen retrieval.

All excised SLNs were sent to the Pathology Department separately from other materials and were examined in detail. After harvesting, two different senior pathologists examined the specimens and lymph nodes by using a standard pathologic method. If the SLNs were negative after routine $\mathrm{H} \& \mathrm{E}$ staining, they were sectioned at $150-\mu \mathrm{m}$ intervals and examined at four or five levels by $\mathrm{H} \& \mathrm{E}$ staining and by immunohistochemistry using a pan-cytokeratin antibody (1:50, mouse antihuman cytokeratin monoclonal antibody, clone AE1/AE3, IgG1-kappa, M3515; DakoCytomation, Carpinteria, CA, USA). The remaining non-SLNs underwent an identical histopathological workup and were examined with serial 

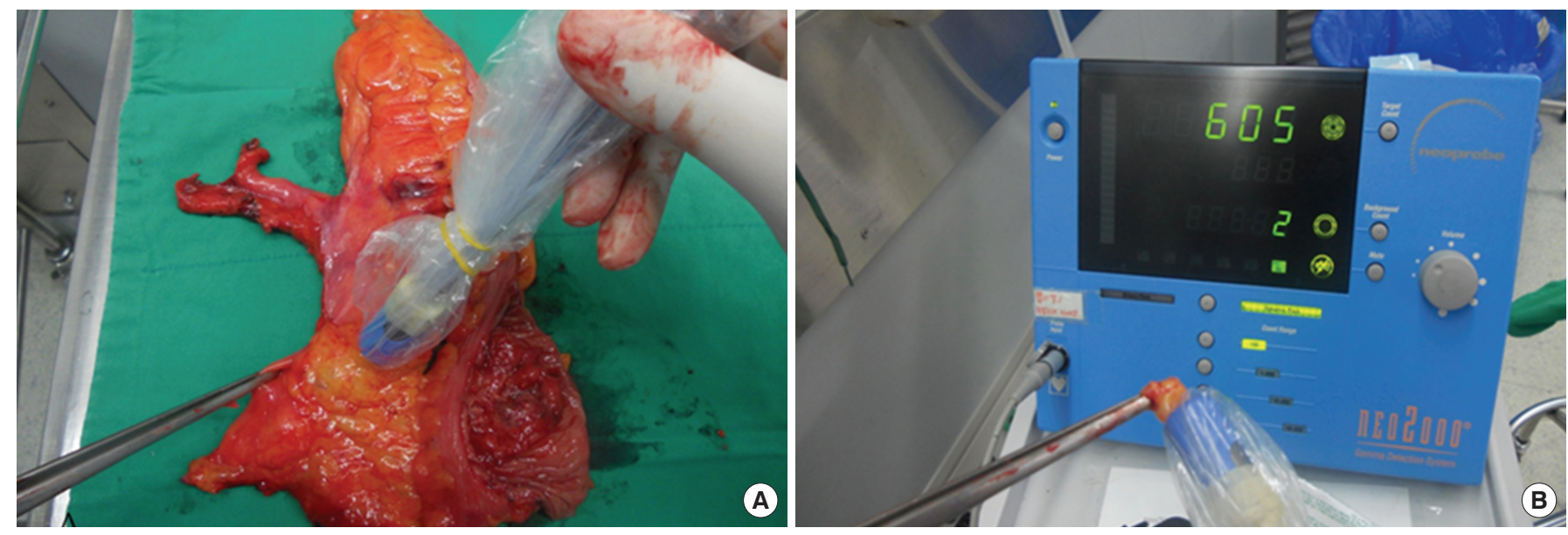

Fig. 2. Sentinel node navigation on the back table. (A) A gamma-radiation probe-guided sentinel node mapping in patients with rectal cancer. (B) Bench work at a separate back table to pick up each hot node with radioactivity ten times that of background.

section $\mathrm{H} \& \mathrm{E}$ staining and cytokeratin immunohistochemical staining.

We classified lymph nodes according to the Japanese classification of colorectal carcinoma. The Japanese criteria define the nodes at the origin of the inferior mesenteric artery (IMA; station 253) as those that lie along the IMA proximal to the origin of the left colic artery. They define the inferior mesenteric trunk nodes (station 252) as those that lie along the IMA from distal to the origin of the left colic artery to the bifurcation of the superior rectal artery and the pararectal nodes (station 251) as those that lie along the superior rectal artery. They are categorized as sigmoid nodes (station 242) when they are located along the sigmoid artery between the IMA and the marginal artery and as sigmoid mesenteric nodes (station 241) when they are located along the marginal artery between the superior rectal artery and the proximal margin.

In this study, metastases between 0.2 and $2 \mathrm{~mm}$ are referred to as micrometastases, and metastases smaller than $0.2 \mathrm{~mm}$ are referred to as isolated tumor cells. Upstaging is defined as the presence of micrometastases or isolated tumor cells found by using immunohistochemistry for patients with a negative lymph node status after conventional H\&E staining. Only descriptive statistics are used.

\section{RESULTS}

The 20 consecutive patients with rectal cancer who underwent curative total mesonectal excision (TME) between May 2009 and December 2010 were prospectively enrolled. All these patients had no radiologically diagnosed lateral pelvic lymph node metastasis. The median age was 64 years (range, 47 to 80 years), and the ratio of men to women was 10:10. The mean distance from the anal verge was $6.5 \mathrm{~cm}$ (range, 3 to $9 \mathrm{~cm}$ ). The mean tumor size was 3.5 $\mathrm{cm}$ (range, 1.0 to $5.5 \mathrm{~cm}$ ). Tumor features are summarized in Table 1 . Seven patients with low rectal cancer underwent an intersphincteric resection with coloanal anastomosis. Seven of the 20 patients received neo-adjuvant radio-chemotherapy (50 Gy in 25 fractions for five weeks).

In all cases but one, lymphatic flow was visualized on the lymphoscintigraphy (detection rate, 95.0\%) (Table 2). An example of lymphoscintigraphy of an ex-vivo specimen is shown in Fig. 3. In cases of SLN identification, the total number of sentinel nodes retrieved from the surgical specimen was 29 , with a mean number of 1.6 per patient (range, 1 to 4 ). On average, a total of 12.7 lymph nodes (range, 3 to 31 lymph nodes) were harvested per specimen. In three patients, the SLN was the only positive lymph node. In three cases $(3 / 19,15.7 \%)$, hot-uptake with direct lymphatic drainage was detected outside the perirectal region (251 station) (Fig. 3B). The mean time between specimen recovery and ex-vivo mapping was 45 minutes (range, 15 to 75 minutes). There was one patient with failed SLN navigation; this patient had a pT3N1 tumor. He received neo-adjuvant radiotherapy and underwent an intersphincteric resection with coloanal anastomosis. For that patient, seven lymph nodes were harvested with one node metastasis, but no SLN was detected.

Histological analyses showed that 10 of 20 patients had lymph node metastases. In all but one of those, at least one of the SLNs contained tumor cells. In that one patients (false negative case), a tumor cell was found in the sigmoid mesenteric node (station 241), but the SLN was tumor negative. After additional sectioning and staining with pan-cytokeratine, in 2 (20\%) of 10 patients with stage II rectal cancer (with conventional H\&E staining), a micrometastasis $(<2 \mathrm{~mm}$ ) was found (Fig. 4$)$.

\section{DISCUSSION}

Up to $25 \%$ of cases without nodal disease still have recurrence within 5 years. It is thought that relapse could occur in patients who have lymph node metastasis that has not been identified by using a standard histological examination. In current nodal stag- 
Table 1. Clinicopathological characteristics of patients

\begin{tabular}{|c|c|c|c|c|c|c|c|c|c|c|c|c|}
\hline No. & Age (yr) & Sex & $\begin{array}{c}\text { BMl } \\
\left(\mathrm{kg} / \mathrm{m}^{2}\right)\end{array}$ & $\begin{array}{l}\text { Surgical } \\
\text { procedure }\end{array}$ & $\begin{array}{c}\text { Tumor } \\
\text { height } \\
(\mathrm{cm})\end{array}$ & $\begin{array}{l}\text { Pre-OP } \\
\text { CCRT }\end{array}$ & $\begin{array}{l}\text { Tumor } \\
\text { size } \\
(\mathrm{cm})\end{array}$ & $\begin{array}{l}\text { pTN }^{\mathrm{a}} \\
\text { stage }\end{array}$ & $\begin{array}{l}\text { Differen- } \\
\text { tiation }\end{array}$ & $\begin{array}{l}\text { Lymphatic } \\
\text { invasion }\end{array}$ & $\begin{array}{l}\text { Number } \\
\text { of SLN }\end{array}$ & Location of SLN \\
\hline 1 & 67 & $\mathrm{~F}$ & 20.4 & LAR & 9 & None & 4 & T3N1 & Moderate & None & 1 & Para-rectal \\
\hline 2 & 71 & M & 23.3 & LAR & 8 & None & 5 & T3N1 & Moderate & None & 1 & Para-rectal \\
\hline 3 & 47 & $\mathrm{~F}$ & 21.3 & uLAR/CAA & 9 & None & 5.5 & T3N1 & Moderate & Yes & 1 & Para-rectal \\
\hline 4 & 59 & F & 24.2 & LAR & 8 & None & 3.5 & T3NO & Moderate & None & 1 & Para-rectal \\
\hline 6 & 68 & $\mathrm{~F}$ & 24.5 & ULAR/CAA & 4 & None & 1 & T1N0 & Moderate & None & 2 & Para-rectal/inf. mesenteric trunk \\
\hline 7 & 56 & M & 24.9 & LAR & 7 & None & 3 & T2N1 & Moderate & Yes & 2 & Para-rectal/inf. mesenteric trunk \\
\hline 8 & 58 & M & 24.5 & LAR & 7 & Done & 1.5 & T3NO & Moderate & None & 1 & Para-rectal \\
\hline 9 & 65 & M & 22.2 & ULAR/CAA & 3 & Done & 3 & T2N1 & Moderate & None & 2 & Para-rectal/inf. mesenteric root \\
\hline 13 & 60 & M & 19.6 & uLAR/CAA & 4 & Done & 3 & T3NO & Moderate & Yes & 4 & Para-rectal/sigmoid node \\
\hline 14 & 51 & M & 18.9 & LAR & 7 & None & 1.5 & T3N2 & Moderate & None & 1 & Para-rectal \\
\hline 15 & 56 & $\mathrm{~F}$ & 28.4 & LAR & 4 & Done & 4 & T2NO & Well & None & 2 & $\begin{array}{l}\text { Inf. mesenteric trunk/ } \\
\text { sigmoid mesenteric }\end{array}$ \\
\hline 16 & 63 & $F$ & 29.4 & LAR & 8 & None & 1.5 & T1N0 & Moderate & None & 2 & Para-rectal/para-rectal \\
\hline 17 & 60 & M & 22.1 & uLAR/CAA & 3 & Done & 4 & T3N1 & Poor & None & 0 & Not detected \\
\hline 18 & 79 & $\mathrm{~F}$ & 23.5 & uLAR/CAA & 3 & Done & 2.5 & T3N2 & Moderate & None & 1 & Para-rectal \\
\hline 19 & 74 & $\mathrm{~F}$ & 22.6 & LAR & 8 & None & 4 & T3NO & Moderate & None & 1 & Para-rectal \\
\hline 20 & 69 & M & 24.7 & ULAR/CAA & 4 & Done & 1 & T3NO & Moderate & None & 2 & $\begin{array}{l}\text { Inf. mesenteric trunk } \\
\text { inf. mesenteric root }\end{array}$ \\
\hline
\end{tabular}

BMl, body mass index; LPLN, lateral pelvic lymph node; CCRT, combined chemoradiation therapy; LAR, low anterior resection; APR, abdominoperineal resection; CAA, colo anal anastomosis; op, operation; SLN, sentinel lymph node.

aReported according to the sixth edition of the AJCC cancer staging.

ing, only one or two sections of lymph nodes are selected for histopathological evaluation, resulting in a substantial risk of missing metastasis in other parts of the lymph node. Additionally, microscopic error, due to a failure of the conventional histopathological analysis to detect micrometastasis, may contribute to understaging. If the accuracy of staging in patients with colorectal cancer is to be improved, a detailed analysis of lymph nodes recovered from the specimen must be performed. Although the oncologic impact of micrometastais and the risk of recurrence remain to be defined, previous studies have shown that the ultrastaging of lymph nodes with multisectioning, immunohistochemical staining or RT-PCT identifies a subgroup of $\mathrm{pN} 0$ patients with a poorer prognosis [911]. However, it is cost-prohibitive and highly time-consuming to ultrastage all the nodes in a given specimen, especially when the specimen contains multiple nodes. SLN mapping aims to resolve these problems by selecting only a few lymph nodes for detailed histopathological analysis.

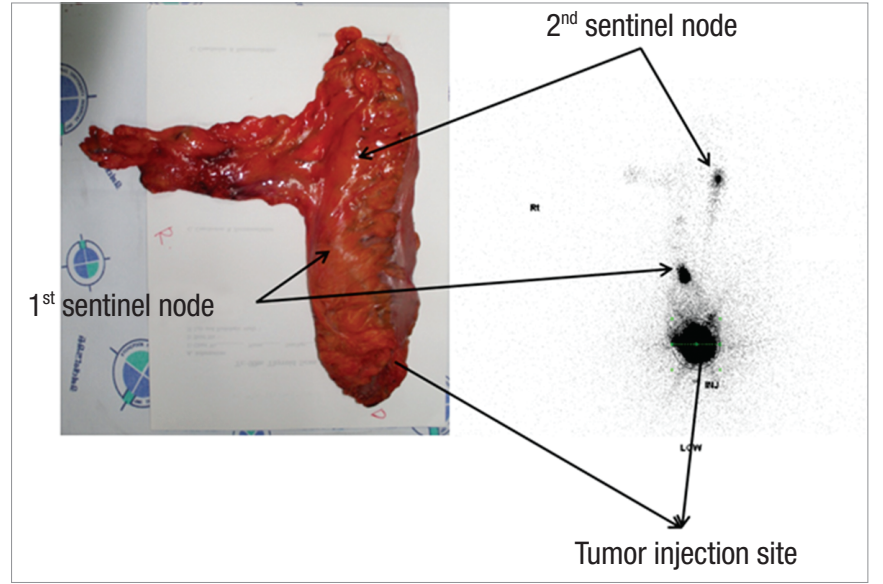

Fig. 3. Postoperative images in patient with drainage to perirectal (station 251) and sigmoid (station 241) lymph nodes. Lymphoscintigraphy 5 hours after injection in patient 13 showed tracer uptake in 2 sentinel nodes. 
Table 2. Results of sentinel lymph node mapping based on injection technique

\begin{tabular}{lcccccc}
\hline No. of cases & No. of SLN & Detection rate (\%) & Accuracy (\%) & Sensitivity (\%) & False-negative rate (\%) & Upstaging rate (\%) \\
\hline 20 & $2.3 \pm 0.9$ & $19 / 20(95.0)$ & $18 / 19(94.7)$ & $8 / 9(88.8)$ & $1 / 9(12.2)$ & $2 / 10(20.0)$ \\
\hline
\end{tabular}

SLN, sentinel lymph node.

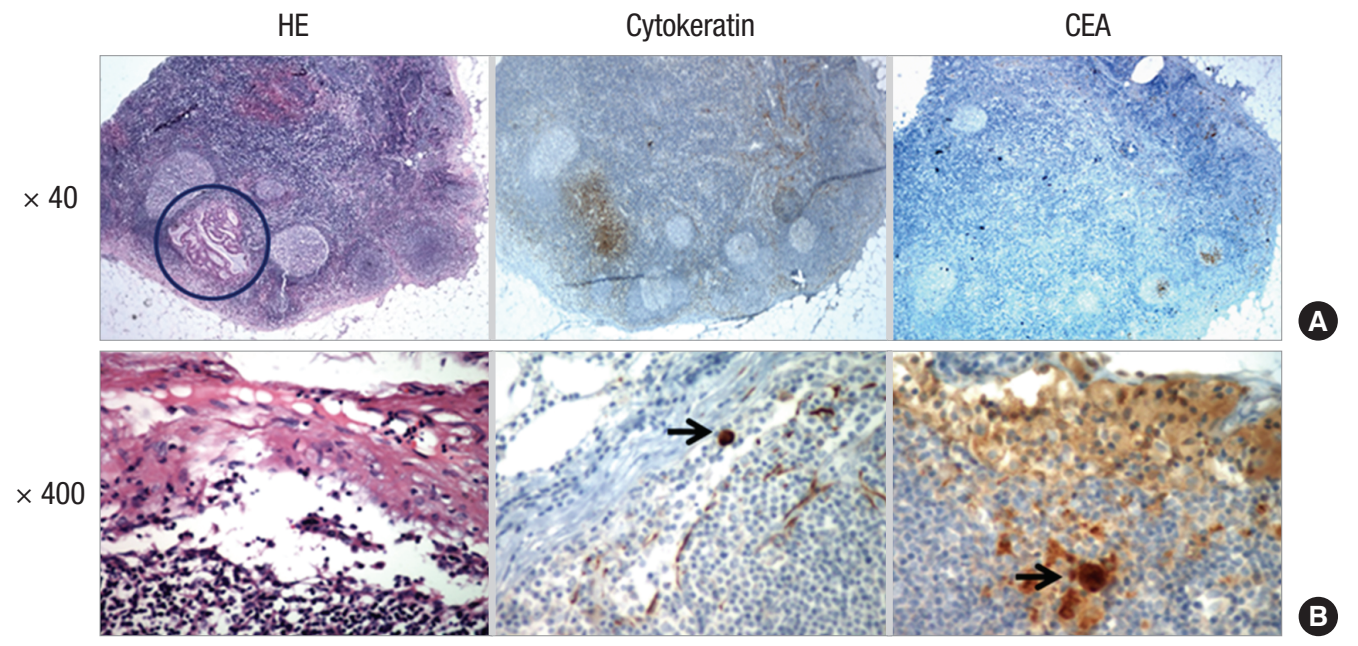

Fig. 4. (A) A micrometastasis (circle) disappears in immunohistochemistry for cytokeratin and carcinoembryonic antigen (CEA) in deeper sections. (B) Isolated tumor cells (arrows) are seen in immunohistochemistry for cytokeratin and CEA, but they are not seen in routine $\mathrm{H} \& \mathrm{E}$ staining.

The current pilot study demonstrated the principle that an ex-vivo lymphoscintigraphy-guided lymph node mapping can provide an acceptable sensitivity and an accurate identification of SLNs in the rectum. Although several studies have described successful SLN identification in cases of colon cancer, with identification rates of 94-100\% and sensitivities ranging from 85 to $100 \%$ [12-14], some authors have suggested that the sentinel lymph node mapping technique might be less applicable for rectal cancer than for colon cancer because they found a higher failure rate for detecting SLNs in rectal cancer $[15,16]$. One of the reasons mentioned is that neoadjuvant chemoradiation obliterating the lymphatic channel or scleroses lymph nodes of the nodal basin draining the primary tumor. A more reasonable explanation might be associated with rectal cancer being located in the infraperitoneal region. Injecting blue dye into the subserosa within the confined pelvic cavity without leakage and navigating the sentinel lymph node intraoperatively during the limited window period (almost 10-20 minutes) even after a complete total mesorectal excision are impractical. In the present study, the authors could reduce injection failure by injecting the dye through transanal submucosal injection immediately before the operation so as to exactly inject the dye under direct vision and to use the physiologic lymphatic vessel as effectively as possible. We were able to obtain promising sensitivity and accuracy by combining the transanal submucosal injection with the back-table work (ex-vivo mapping), which was available for more detailed examination than possible with intraoperative navigation.

A detailed pathological work-up of SLNs may increase the diagnostic accuracy of the examining pathologist, but it also may streamline the tedious process of lymph node evaluation, limiting the number of lymph nodes examined by considering their qualitative fea- tures. Accordingly, in accurate SLN-directed staging of colorectal cancer, the optimal number of regional lymph nodes to be removed remains controversial. According to several previously-published clinical studies, the mean number of excised SLNs has been reported to range from 1 to 7 [17-20]. Morton [21] defined the SLN as the first lymph node that receives afferent lymphatic drainage from a primary tumor, but that definition does not recognize that more than a single SLN may be present. In this study, every node was considered to be a sentinel node when showing either an accumulation of radioactive dye on lymphoscintigraphy or ex-vivo radioactive counts tenfold higher than background activity in the back-table procedures. In our series, the mean window time between the specimen extraction and an SLN mapping was 45 minutes, and the mean number of SLNs was 1.7 per patient (range, 1 to 4 ) with a sensitivity of $90 \%$. Presumably, therefore, a mean number of SLNs of 1.6 might be appropriate not only for identifying lymph-node-harboring malignant metastasis but also for avoiding an increase in the workload associated with a micrometastasis.

Another valuable benefit of the SLN mapping technique could be the ability to recognize aberrant lymphatic drainage, which has been reported in 2 to $29 \%$ of colorectal cancer cases [22-24]. Studying direct lymphatic drainage from rectal cancer may shed light on the unpredictable location of sentinel lymph nodes. In the radical treatment of rectal cancer, metastasis to the sigmoid mesenteric (station 241) and the sigmoid lymph (station 242) nodes should be considered as an aberrant metastasis, yet the status of the sigmoid mesenteric or the sigmoid lymph nodes is not well defined. This may be due to the technical difficulties in dissecting or sampling all sigmoid lymph nodes and in mobilizing the splenic flexure. Several studies have reported on the outcome of a J-pouch res- 
ervoir using a sigmoid colon segment for an improved functional outcome after TME. Of interest, we found that radiocolloid particles were directly accumulated around the sigmoid mesenteric or sigmoid lymph node station in 3 of 19 patients (15.7\%). The findings obtained in this study are in agreement with previous findings that not only the root of the inferior mesenteric lymph node, but a sizable part of the sigmoid colon with the sigmoid mesenteric lymph node should be included in the extent of dissection in the subset of patients with rectal cancer [25].

The present study revealed micrometastases or isolated tumor cells in the SLNs of $20.0 \%$ of patients initially classified as pN0 based on a conventional examination. Therefore, our results confirm the findings of early studies using SLN mapping [7, 20, 26]. However, these results did not influence adjuvant treatment decisions in our clinical setting. Lymphatic mapping in the present trial was considered investigational, and our oncologists performed adjuvant treatment according to conventional guidelines. Until now, the influence of lymphatic micrometastases on cancer prognosis has not been clearly established. Although some studies have shown that micrometastases can adversely affect disease outcomes, several other studies have been unable to show such a correlation. A recent multicenter trial that represents the first prospective evaluation of the prognostic impact of micrometastases detected a direct correlation between the presence of a micrometastasis and diseasefree survival [12]. However, further additional, randomized, prospective trials and long-term follow-up studies are warranted to assess the prognostic value of ultrastaging. This is because the follow ups in the previous literature were relatively short and because evidence supporting the trend for administration of adjuvant chemotherapy to patients with micrometastasis exists.

The strengths of this study include the whole evaluation of micrometastasis in both SLNs and non-SLNs. The complete analysis made it possible to assess the actual upstaging rates and false negative rates. The main limitation of the study is the mixed cohorts, including preoperative chemoradiation and non-radiation subsets. Further study with a homogeneous study group is needed, taking into consideration the impact of preoperative radiation on the lymphatic pathway. The relatively low rectal location of the target lesion is another drawback of the current study. We mainly have to deal with extraperitoneal tumors due to injection with a transanal approach under direct vision. An evaluation of an endoscopic mapping protocol of procedures that integrates preoperative tattooing and dye injection via colonoscopy is now in progress so that it can be reported in the future.

In conclusion, SLN identification and sampling after preoperative transanal injection with detection on lymphoscintigraphy is surgically feasible and safe in patients with extraperitoneal rectal cancer. Preliminary data suggest that SLNs from the rectal cancer are mainly localized in the pararectal region, but aberrant nodes receiving direct drainage from the rectal cancer had developed in $15.7 \%$ of the cases. To draw more conclusive scientific conclusions, we must wait to increase the number of cases submitted to this procedure protocol and to increase the duration of follow-up evaluation.

\section{CONFLICT OF INTEREST}

No potential conflict of interest relevant to this article was reported.

\section{ACKNOWLEDGEMENTS}

This study was supported by a grant of the Korea Healthcare Technology R\&D Project, Ministry of Health \& Welfare, Republic of Korea (study ID: A090907).

\section{REFERENCES}

1. Moertel CG, Fleming TR, Macdonald JS, Haller DG, Laurie JA, Tangen CM, et al. Fluorouracil plus levamisole as effective adjuvant therapy after resection of stage III colon carcinoma: a final report. Ann Intern Med 1995;122:321-6.

2. Efficacy of adjuvant fluorouracil and folinic acid in B2 colon cancer. International Multicentre Pooled Analysis of B2 Colon Cancer Trials (IMPACT B2) Investigators. J Clin Oncol 1999;17:1356-63.

3. Moertel CG, Fleming TR, Macdonald JS, Haller DG, Laurie JA, Tangen CM, et al. Intergroup study of fluorouracil plus levamisole as adjuvant therapy for stage II/Dukes' B2 colon cancer. J Clin Oncol 1995;13:2936-43.

4. Cabanas RM. An approach for the treatment of penile carcinoma. Cancer 1977;39:456-66.

5. Saha S, Monson KM, Bilchik A, Beutler T, Dan AG, Schochet E, et al. Comparative analysis of nodal upstaging between colon and rectal cancers by sentinel lymph node mapping: a prospective trial. Dis Colon Rectum 2004;47:1767-72.

6. Bilchik AJ, DiNome M, Saha S, Turner RR, Wiese D, McCarter M, et al. Prospective multicenter trial of staging adequacy in colon cancer: preliminary results. Arch Surg 2006;141:527-33.

7. Wood TF, Saha S, Morton DL, Tsioulias GJ, Rangel D, Hutchinson $\mathrm{W}$ Jr, et al. Validation of lymphatic mapping in colorectal cancer: in vivo, ex vivo, and laparoscopic techniques. Ann Surg Oncol 2001;8:150-7.

8. Hutteman M, Choi HS, Mieog JS, van der Vorst JR, Ashitate Y, Kuppen PJ, et al. Clinical translation of ex vivo sentinel lymph node mapping for colorectal cancer using invisible near-infrared fluorescence light. Ann Surg Oncol 2011;18:1006-14.

9. Adell G, Boeryd B, Franlund B, Sjodahl R, Hakansson L. Occurrence and prognostic importance of micrometastases in regional lymph nodes in Dukes' B colorectal carcinoma: an immunohistochemical study. Eur J Surg 1996;162:637-42.

10. Noura S, Yamamoto H, Ohnishi T, Masuda N, Matsumoto T, Takayama $\mathrm{O}$, et al. Comparative detection of lymph node micrometastases of stage II colorectal cancer by reverse transcriptase polymerase chain reaction and immunohistochemistry. J Clin Oncol 2002;20:4232-41.

11. Palma RT, Waisberg J, Bromberg SH, Simao AB, Godoy AC. Mi- 
crometastasis in regional lymph nodes of extirpated colorectal carcinoma: immunohistochemical study using anti-cytokeratin antibodies AE1/AE3. Colorectal Dis 2003;5:164-8.

12. Bilchik AJ, Hoon DS, Saha S, Turner RR, Wiese D, DiNome M, et al. Prognostic impact of micrometastases in colon cancer: interim results of a prospective multicenter trial. Ann Surg 2007;246:56875.

13. Kelder W, Braat AE, Karrenbeld A, Grond JA, De Vries JE, Oosterhuis JW, et al. The sentinel node procedure in colon carcinoma: a multi-centre study in the Netherlands. Int J Colorectal Dis 2007; 22:1509-14.

14. Saha S, Sehgal R, Patel M, Doan K, Dan A, Bilchik A, et al. A multicenter trial of sentinel lymph node mapping in colorectal cancer: prognostic implications for nodal staging and recurrence. Am J Surg 2006;191:305-10.

15. van Schaik PM, van der Linden JC, Ernst MF, Gelderman WA, Bosscha K. Ex vivo sentinel lymph node "mapping" in colorectal cancer. Eur J Surg Oncol 2007;33:1177-82.

16. Braat AE, Oosterhuis JW, Moll FC, de Vries JE, Wiggers T. Sentinel node detection after preoperative short-course radiotherapy in rectal carcinoma is not reliable. Br J Surg 2005;92:1533-8.

17. Cserni G, Vajda K, Tarjan M, Bori R, Svebis M, Baltas B. Nodal staging of colorectal carcinomas from quantitative and qualitative aspects. Can lymphatic mapping help staging? Pathol Oncol Res 1999;5:291-6.

18. Feig BW, Curley S, Lucci A, Hunt KK, Vauthey JN, Mansfield PF, et al. A caution regarding lymphatic mapping in patients with colon cancer. Am J Surg 2001;182:707-12.

19. Gandy CP, Biddlestone LR, Roe AM, O'Leary DP. Intra-operative injection of Patent Blue V dye to facilitate nodal staging in colorectal cancer. Colorectal Dis 2002;4:447-9.

20. Joosten JJ, Strobbe LJ, Wauters CA, Pruszczynski M, Wobbes T, Ruers TJ. Intraoperative lymphatic mapping and the sentinel node concept in colorectal carcinoma. Br J Surg 1999;86:482-6.

21. Morton DL. Current managment of malignant melanoma. Ann Surg 1990;212:123-4.

22. Saha S, Wiese D, Badin J, Beutler T, Nora D, Ganatra BK, et al. Technical details of sentinel lymph node mapping in colorectal cancer and its impact on staging. Ann Surg Oncol 2000;7:120-4.

23. Tsioulias GJ, Wood TF, Morton DL, Bilchik AJ. Lymphatic mapping and focused analysis of sentinel lymph nodes upstage gastrointestinal neoplasms. Arch Surg 2000;135:926-32.

24. Waldman SA, Cagir B, Rakinic J, Fry RD, Goldstein SD, Isenberg $\mathrm{G}$, et al. Use of guanylyl cyclase $\mathrm{C}$ for detecting micrometastases in lymph nodes of patients with colon cancer. Dis Colon Rectum 1998;41:310-5.

25. Park IJ, Choi GS, Lim KH, Kang BM, Jun SH. Metastasis to the sigmoid or sigmoid mesenteric lymph nodes from rectal cancer. Ann Surg 2009;249:960-4.

26. Bembenek AE, Rosenberg R, Wagler E, Gretschel S, Sendler A, Siewert JR, et al. Sentinel lymph node biopsy in colon cancer: a prospective multicenter trial. Ann Surg 2007;245:858-63. 